\title{
Integralidade no cuidado a usuários com lesões de pele: percepção dos profissionais da atenção primária
}

\author{
Integrality in the care of users with skin lesions: perception of primary care professionals
}

\author{
Andrea Gonçalves Bandeiraa , Caroline Trennepohlb, Heloísa Maria Reckziegel Belloc, \\ Adolfo Pizzinato ${ }^{d}$ \\ a Enfermeira. Doutoranda do Programa de Pós-Graduação em Enfermagem da Universidade Federal do Rio Grande do Sul (UFRGS). Professora Assistente do \\ Curso de Graduação em Enfermagem da Pontifícia Universidade Católica do Rio Grande do Sul (PUCRS). \\ b Enfermeira. Especialista em Saúde da Família e Comunidade pelo Programa de Residência Multiprofissional em Saúde da Família e Comunidade (PREMUS/2009) da PUCRS. \\ c Enfermeira. Mestre em Enfermagem pela UFRGS. Professora Assistente da Faculdade de Enfermagem, Nutrição e Fisioterapia da PUCRS. \\ d Psicólogo. Doutor em Psicologia da Educação pela Universitat Autònoma de Barcelona. Professor Adjunto Faculdade de Psicologia da PUCRS.
}

RESUMO I Objetivo: Conhecer a percepção de profissionais de enfermagem inseridos na Atenção Primária em Saúde sobre aplicação do princípio da integralidade no cuidado a usuários com lesões de pele.

Materiais e Métodos: Abordagem qualitativa teve coleta dos dados realizada em diferentes serviços de Porto Alegre, em 2012. A população compreendeu sete enfermeiros e 14 técnicos de enfermagem, que atuam em unidades de saúde que foram campo de prática de um Programa de Residência Multiprofissional em Saúde da Família e Comunidade. Para a coleta de dados foi realizada uma entrevista semiestruturada, as entrevistas foram agendadas previamente conforme a disponibilidade dos profissionais, individual, com duração média de 20 minutos.

Resultados: Foram organizados em torno do eixo Integralidade no cuidado de lesões de pele e deste emergiram duas categorias: (1) integralidade - conhecimento versus distanciamento; (2) o cuidado integral a usuários com lesão de pele - da teoria à prática.

Conclusão: Os participantes mostraram-se protagonistas ativos da integralidade neste campo de prática, apesar das críticas às dificuldades do processo de trabalho em saúde e os desafios encontrados.

Palavras-chave: atenção primária à saúde; enfermagem em saúde comunitária; cuidados de enfermagem; assistência integral a saúde; pesquisa qualitativa.

\section{ABSTRACT}

Objective: To know the perception of nursing professionals inserted in Primary Health Care on the application of the principle of integrality in the care of users with skin lesions.

Materials and Methods: This is a study with a qualitative approach. Data collection was performed in different services of Porto Alegre in 2012. The population comprised seven nurses and 14 nursing technicians, who work in health care units that were also the field of practice for a Multi-professional Residency Program in Family and Community Health. For the data collection, a semi-structured interview was conducted. The interviews were previously scheduled according to the availability of the individual professionals, with an average duration of 20 minutes.

Results: Integrality in the care of skin lesions were organized around two categories: (1) integrality - knowledge versus distancing; (2) comprehensive care for users with skin lesions - from theory to practice.

Conclusion: The participants were active protagonists of integrality in this field of practice, despite the criticisms to the difficulties of the health work process and the challenges encountered.

Keywords: primary health care; community health nursing; nursing care; health care; qualitative research.

\section{Correspondência:}

ANDREA GONCALVES BANDEIRA

Rua Lobo da Costa, 291, apto 302 - Azenha

90050-110 Porto Alegre, RS, Brasil

E-mail: deiabandeira@hotmail.com 


\section{INTRODUÇÃO}

Nas décadas de 1970 e 1980 as estratégias de atenção à saúde no Brasil nem sempre visavam o bem-estar geral da população. As políticas de saúde, muitas vezes, refletiam apenas as urgências do momento vivido, a economia vigente e os ideais de assistência das classes dominantes. Frente a isto, um movimento social e intelectual, denominado sanitarista, surgiu com a finalidade de buscar mudanças nas políticas públicas de saúde 1 .

Este movimento teve como marco histórico a VIII Conferência Nacional de Saúde (1986), seguido da promulgação da nova Constituição Brasileira (1988) que estabeleceu a seguinte diretriz: "Saúde é direito de todos e dever do Estado". Nesse processo foi idealizado o Sistema Único de Saúde (SUS) que tem por base os princípios doutrinários da universalidade, equidade e integralidade ${ }^{2,3}$.

A integralidade da atenção é meta que precisa ser trabalhada em várias dimensões para que seja alcançada da forma mais completa possível ${ }^{4}$. Em seu plano conceitual é uma diretriz chave do sistema sanitário brasileiro, prioriza as ações preventivas sem desconsiderar as curativas, abrangendo o individual e o coletivo, no intuito de melhorar a qualidade de vida da população $0^{5}$. O cuidado a usuários com lesões de pele, por exemplo, foi tradicionalmente visto como procedimento concreto e objetivo, dificultando sua significação como ato de cuidado integral.

Em consonância com a prerrogativa de integralidade, eixo transversal do cuidado em saúde entende-se que o cuidado com lesões de pele, na atenção primária em saúde, vai além de um mero procedimento, pois é visto como porta de entrada para que o profissional possa conhecer o usuário e identificar suas necessidades, a fim de prestar cuidado qualificado, efetivo e integral 6 .

Qualquer quebra da continuidade da pele é chamada de lesão de pele ${ }^{7}$. As lesões de pele acometem a população de forma geral, sendo motivo de procura frequente para atendimento ambulatorial de enfermagem na Atenção Primária em Saúde (APS). Embora não haja dados significativos no Brasil sobre a incidência e prevalência do tratamento de lesões de pele agudas ou crônicas, o impacto psíquico, social e econômico da cronificação das lesões, em especial úlceras crônicas de pés e pernas, representa a segunda causa de afastamento do trabalho no Brasil ${ }^{8}$.

No Brasil, estudo aponta a falta de sistematização da assistência ao usuário com lesões de pele, principalmente quando se trata de úlceras venosas na Atenção Primária à Saúde (APS) ${ }^{9}$. Poucos municípios adotam protocolos clínicos que direcionem ações de cuidados voltadas à prevenção e tratamento dessas lesões. Esta situação pode trazer implicações aos usuários em relação ao tempo de cicatrização, refletindo em sua qualidade de vida e ainda onerando financeiramente o sistema público de saúde com gastos desnecessários ${ }^{10,11}$.

Frente ao exposto, este estudo buscou responder a seguinte questão de pesquisa:

Qual a percepção dos profissionais da Atenção Primária em saúde sobre a aplicação do princípio da integralidade no cuidado a usuários com lesões de pele? Assim, teve como objetivo conhecer a percepção dos profissionais de enfermagem, inseridos na Atenção Primária em Saúde, sobre a aplicação do princípio da integralidade no cuidado a usuários com lesões de pele.

\section{MATERIAIS E MÉTODOS}

O presente estudo configurou-se em abordagem de tipo qualitativo, descritivo e exploratório ${ }^{12,13}$. Os participantes foram os profissionais de enfermagem que atuam em cinco unidades de saúde do Distrito Sanitário Leste/Nordeste de Porto Alegre e campo de prática do Programa de Residência Multiprofissional em Saúde da Família e Comunidade da Pontifícia Universidade Católica do Rio Grande do Sul (PREMUS/PUCRS). Destas, duas são Unidades Básicas de Saúde, e três Unidades de Saúde com Estratégia de Saúde da Família. O grupo estudado foi composto por profissionais que contemplaram os critérios de inclusão: estarem locados nestes serviços há pelo menos um ano, devido o conhecimento acerca do serviço e vinculação aos usuários que necessitam do cuidado de lesões de pele; que prestam diretamente este cuidado ao usuário. A constituição da amostra do estudo foi por conveniência.

Participariam do estudo 21 profissionais de enfermagem, sendo sete enfermeiros e 14 técnicos ou auxiliares de enfermagem. Desta amostra cinco enfermeiros e oito técnicos de enfermagem são das Unidades com ESF e dois enfermeiros e seis técnicos de enfermagem de UBS. Contudo, desta amostra 12 profissionais foram excluídos por não contemplar os critérios de inclusão ou não ter interesse em participar da pesquisa.

A coleta de dados foi realizada entre abril e maio de 2012. As entrevistas foram agendadas previamente conforme a disponibilidade dos profissionais. Para a coleta de dados foi realizada uma entrevista semiestruturada ${ }^{12}$, individual, com duração média de 20 minutos. No momento da entrevista se fez necessário o uso do gravador.

O presente estudo foi aprovado pelos Comitês de Ética em Pesquisa da Secretaria Municipal de Saúde de Porto Alegre parecer no 001.004465.11.3, e da Pontifícia Universidade Católica do Rio Grande do Sul no 11/05359. Para a garantia do anonimato dos sujeitos do estudo foram 
atribuídos nomes fictícios independente do sexo, a fim de mesclar os sujeitos, visto que a maioria dos participantes era do sexo feminino. Às unidades de saúde também foram atribuídas siglas e números para identificação ${ }^{14}$.

Para análise dos dados foi realizada a transcrição das entrevistas e após sucessivas leituras os dados foram organizados em eixos temáticos ${ }^{12}$. Para auxiliar na categorização fez-se o uso do programa Atlas/ti, que foi desenvolvido na Alemanha na década de 90, e tem como uma de suas raízes a análise de conteúdo. É uma ferramenta de ajuda ao intérprete que opera em dois níveis de constante fluxo, envolve atividades de encontro e segmentação de texto, codificação e escrita de comentários, facilitando assim a recuperação da informação e atividades de modelagem gráfica e conexão de todos os elementos da unidade ${ }^{15}$.

$\mathrm{Na}$ pesquisa qualitativa os desafios são dar sentido a uma quantidade massiva de dados, reduzir o volume de informação, identificar pautas significativas e construir um marco para comunicar a essência do que revelam os dados. Esta ideia de redução de dados é um dos princípios fundamentais da pesquisa qualitativa. Ainda que a pesquisa tenha utilizado o Atlas/Ti o conceito de codificação empregado é mais próximo ao da pesquisa qualitativa de caráter discursivo, ou seja, a codificação se processa mediante o desenvolvimento intencional e o "etiquetado" de conceitos no texto, ambas de relevância potencial para o problema que está sendo estudado.

A codificação axial foi o passo seguinte na análise, posto que, uma vez criados códigos - tanto específicos como genéricos (categorias e subcategorias) - procede-se à identificação de relações entre os mesmos. O programa informático oferece a possibilidade de relacionar códigos entre si de forma simples, permitindo ao analista escolher os tipos de relações previamente definidos no programa ou criando as suas próprias relações ${ }^{16}$.

\section{RESULTADOS}

Os resultados do estudo foram organizados em torno do eixo central que é a própria Integralidade no cuidado de lesões de pele, categoria que emergiu como a central nos conteúdos colhidos nas entrevistas ( $46 \%$ das verbalizações selecionadas). Os demais marcadores temáticos são desdobramentos deste: Integralidade - Conhecimento versus Distanciamento (28\%); O cuidado integral a usuários com lesão de pele - da teoria à prática (26\%). Como na epistemologia qualitativa, tal representação numérica é praticamente irrelevante, optouse por definir a construção das categorias em sua relação com os dados coletados na própria discussão, como costuma ocorrer nos estudos qualitativos. À seguir se apresentam os marcadores temáticos e se exemplificam suas construções na pesquisa, tanto no diálogo com os autores em que nos apoiamos como, especialmente, nas narrativas dos/das participantes da pesquisa.

\section{Integralidade: conhecimento versus distanciamento}

Quando indagados sobre o que entendiam em relação ao princípio da integralidade do SUS os profissionais de enfermagem, que mostraram conhecimento sobre o mesmo, se remeteram a ver o usuário como um todo, de uma forma global e holística, indo ao encontro com os conceitos supracitados. Discurso este que fica explicito na fala abaixo:

"Eu entendo como integralidade quando tu atendes um paciente e tu não enxerga só aquela feridinha ou só aquela queixa principal, inicial e sim o paciente como um todo." (Marcelo - ENF Unidade 3)

Identificou-se, nos discursos de alguns enfermeiros, formas de como conheceram o princípio da integralidade do SUS, considerando este saber como algo inerente a sua profissão, personalidade ou formação:

"Eu acho que a enfermeira já tem um dom natural [...] Porque a nossa formação é voltada para a integralidade do cuidado." (Lara - ENF Unidade 5)

Outros enfermeiros atribuem seu conhecimento devido à prática diária nos serviços de saúde:

"O que eu sei é do que eu li e vi depois que eu saí da faculdade, porque quando eu estudei não falavam em SUS, em integralidade [...] Não existia isso." (Letícia ENF Unidade 2)

Entretanto, outros profissionais atribuem o conhecimento sobre integralidade à sua inserção na Atenção Primária em Saúde, reiterando que este conceito não estava presente em sua prática profissional anterior no âmbito hospitalar:

"Quando eu entrei aqui eu não tinha noção porque eu trabalhava em hospital [...]." (Fernando - TE Unidade 3)

Destaca-se, neste mesmo contexto, que outros profissionais apresentaram dúvidas em relação ao que seria esse princípio, confundindo muitas vezes com integração da equipe e trabalho multiprofissional.

"Olha, exatamente só sobre isso eu não ouvi muito assim [...]." (Frederico - TE Unidade 2)

"É trabalhar entre as equipes, né? se eu não to enganada integralidade... é que eu faço uma confusão às vezes entre integralidade e multi [...]." (Francis - TE Unidade 2) 


\section{O cuidado integral a usuários com lesão de pele: da teoria a prática}

Quando questionado aos profissionais de enfermagem se conseguem aplicar na prática a integralidade ao cuidado ao usuário portador de lesões de pele e, como se dá essa prática, nos deparamos com as seguintes falas:

"É [...] às vezes até se consegue, dependendo da gravidade de cada paciente, né?" (Flavio - TE Unidade 3)

"Em relação ao cuidado integral [...] A gente conversa vê o tipo de ferimento, vê como é que aconteceu se estava em casa [...] Então a gente tenta ver todo o lado, onde mora, quais as condições, o que aconteceu, o que provocou como poderia ser evitado [...]." (Fabricio - TE Unidade 1)

Em sua fala o técnico de enfermagem Flávio traz que colocar em prática o princípio da integralidade em seu dia a dia estaria atrelado à gravidade do caso, e dependendo também dos recursos disponíveis na APS, deixando implícito que nos casos mais graves há dificuldade em colocá-lo em prática.

Essa situação ficou evidente nas entrevistas, quando indagamos aos profissionais sobre a prática da integralidade, e os discursos em destaque foram todos de auxiliares e técnicos de enfermagem, reforçando que este cuidado, principalmente ao usuário com lesão de pele, é realizado em grande parte por eles. Os enfermeiros, no contexto desta pesquisa, mostraram ter um papel mais teórico, pois detém o conhecimento sobre o conceito da integralidade, mas essa prática encontra-se um pouco distante do seu processo de trabalho, aparecendo na maioria das vezes como uma atividade de supervisão e avaliação.

"[...] Eu exerço muitas atividades assistenciais, mas mais a coordenação, então eu fujo um pouco [...] claro que quando tem uma situação chama, ah enfermeira vem aqui dá uma avaliada." (Laura - ENF Unidade 4)

\section{DISCUSSÃO}

A seguir discutem-se os resultados das categorias encontradas, apoiando-se na literatura da área e, especialmente nas verbalizações dos participantes, nas três categorias centrais encontradas.

No que diz respeito à integralidade vem sendo reforçada desde a década de 90 com o surgimento do Programa de Saúde da Família (PSF) em 1994 e com a Política Nacional de Humanização do SUS, o Humaniza SUS ${ }^{17,18}$. Desde então, a integralidade no cuidado se tornou mais presente nas práticas diárias dos profissionais da saúde.
Neste mesmo contexto, a integralidade consistiria em uma resposta ampla ao sofrimento do paciente que procura o serviço de saúde, mantendo a atenção para que as estratégias de cuidado não sejam reduzidas à ferida ou sistema biológico deste, pois tal redução criaria lacunas tanto no entendimento global da situação que se apresenta quanto às possíveis angústias ou sofrimentos gerados pela ferida ou até mesmo prévios a ela e que podem ter inclusive contribuído no estado físico apresentado ${ }^{19}$. Neste sentido corrobora-se trazendo que a integralidade busca a totalidade do sujeito, evitando a fragmentação e o reducionismo ${ }^{20}$.

Atribui-se a visão mais ampla destes profissionais e a familiaridade com o tema proposto, à sua inserção em Unidades de Saúde com Estratégia de Saúde da Família, visto o objetivo desta de reorientar a APS, buscando a centralidade de suas ações na integralidade da atenção e do cuidado à família e comunidades ${ }^{19}$. Na Estratégia de Saúde da Família (ESF) a equipe de saúde deve conhecer as famílias de seu território de abrangência, identificar os problemas de saúde e as situações de riscos existentes na comunidade, elaborar programas de atividades para enfrentar os determinantes do processo saúde/doença, desenvolver ações educativas e intersetoriais relacionadas com os problemas de saúde identificados e prestar assistência integral às famílias sob sua responsabilidade ${ }^{21,22}$. A inserção no serviço faz com que o profissional se aproprie da realidade do local e do modelo de assistência do mesmo, fazendo com que ele busque informações acerca dos mesmos e suas práticas diárias acabam caracterizando um aprendizado formativo. Percebe-se também que a busca pelo conhecimento depende da motivação pessoal de cada profissional e do local onde estão inseridos.

Os discursos demonstram que ainda podem existir lacunas nos currículos dos cursos de graduação e técnicos de enfermagem, pois até bem pouco tempo estavam completamente voltados para a formação para atender à área hospitalar, ficando a APS como um nível de atenção sem grande importância, por não deter serviços de alta complexidade, mesmo ela sendo considerada a porta de entrada do SUS. O fato ainda pode estar associado à formação profissional, predominantemente baseada em uma atenção hospitalar centrada na doença e no médico que limita o cuidado às práticas pontuais e curativas. Nesta lógica, um dispositivo essencial para mudança no processo de trabalho é a preparação das equipes, com visões mais integralizadoras e novas competências voltadas para um perfil adequado ao modelo proposto ${ }^{22}$.

A enfermagem, nessa construção interdisciplinar pode ser chamada a assumir seu núcleo de competência e responsabilidade em uma posição chave para a elaboração de estratégias de cuidado. Esse cuidado teria como desafio 
considerar também as subjetividades individuais e coletivas. Mediante isso, a área da enfermagem manifesta potência para transitar em diferentes campos de conhecimento, pois a profissão tem como foco a pessoa a ser cuidada, podendo assim estabelecer, mais intensivamente, canais de interlocução junto a outros agentes, com a intenção de buscar tecnologias necessárias à assistência, mantendo relações com a equipe e, também, com a família, inclusive atuar no processo de transformação da realidade, desta forma apreendendo e colocando em prática a integralidade ${ }^{22,23}$.

Justifica-se assim o aprendizado dos profissionais durante as práticas de trabalho, pois se percebe que, apesar do discurso da maioria dos participantes caracterizarem uma aproximação ao tema e um conhecimento significativo acerca do conceito da integralidade, cabe ressaltar que alguns profissionais se mostraram distantes, não sabendo do que se tratava, apresentando um desconhecimento em relação ao SUS, mesmo estando todos inseridos na APS pública há muitos anos.

Já a prática de cuidados a usuários com lesão de peles é um desafio que requer conhecimento específico, habilidade e abordagem holística. A enfermagem, na maioria das vezes é a responsável pelo cuidado direto da lesão de pele, porém, cabe lembrar que não se trata somente de um procedimento técnico pontual, sendo necessário que o profissional veja além dela e contemple o usuário como um todo, de acordo com as prerrogativas da integralidade, um dos eixos orientativos de toda ação em saúde dentro do SUS.

Os estudos sobre tratamento de lesões de pele recebem grande destaque nas publicações de enfermagem, demonstrando que a responsabilidade da prevenção e do tratamento destas vem sendo atribuída ao enfermeiro, devendo ele avaliar a lesão e prescrever o tratamento mais adequado, além de orientar e supervisionar a equipe de enfermagem na execução do curativo ${ }^{24}$.

A integralidade do cuidado está presente no encontro, na conversa e na atitude do profissional que procura reconhecer para além das demandas explícitas do usuário que busca o serviço de saúde. Desta forma a integralidade existe em ato, sendo reconhecida nas práticas que valorizam o cuidado e que têm em suas concepções a idéia de considerar o usuário como sujeito a ser atendido e respeitado em suas demandas e necessidades ${ }^{4,24}$.

Frente à ascensão de muitos dos participantes à gestão de seus serviços, vê-se que os enfermeiros acabam se distanciando um pouco do cuidado direto com o usuário ou que ele acaba desenvolvendo muitas vezes atividades voltadas para o aspecto curativo em detrimento de sua função preventiva e educativa, visto que desta forma o tempo a ser ocupado seria menor.
Conclui-se que as funções que os enfermeiros exercem em seu trabalho são, em grande parte, na área administrativa ou técnica, ficando de lado ou deixando a desejar uma atuação mais intensa voltada para a prevenção, para a promoção e para a humanização da assistência. Logo, entende-se que é necessário que o enfermeiro na APS perceba e reconheça que essas competências são intrínsecas ao seu cotidiano, e que cabe a cada um tentar conciliar a assistência e a coordenação.

Apesar de consonante com a decisão epistemológica adotada e, portanto, sem nenhuma pretensão de generalização dos dados obtidos e analisados, este estudo possibilitou um conhecimento maior acerca da percepção dos profissionais de enfermagem da APS de Porto Alegre na integralidade do cuidado a usuários com lesão de pele. Além disso, propiciou um entendimento mais complexo dos processos dos processos de trabalho na área do tratamento de lesões de pele, abrangendo desde o conhecimento sobre o princípio da integralidade, o distanciamento do mesmo das práticas de cuidado possíveis no contexto estudado, bem como os desafios encontrados pelos profissionais da enfermagem (tanto enfermeiros e técnicos de enfermagem). Encontrou-se que os enfermeiros apresentam maior domínio sobre a teoria do princípio da integralidade. Os técnicos de enfermagem trouxeram a integralidade nas suas práticas diárias, além de elucidarem as formas utilizadas para praticá-la, mesmo que suas definições não fossem tão próximas às teoricamente consensuadas.

Identificaram-se ainda alguns profissionais, inseridos em Unidades sem ESF, que não possuíam conhecimento significativo acerca da temática abordada - independentemente da formação - evidenciando que ainda há uma distância entre os modelos assistências e de organização do processo de trabalho, refletindo na atenção ao usuário. Quanto ao cuidado às lesões de pele, os técnicos e auxiliares de enfermagem são os que mais o realizam e cabem aos enfermeiros mais atividades de supervisão e avaliação dessas práticas. Considera-se também que neste momento do cuidado ao usuário com lesões de pele existe a oportunidade de levantamento de demandas desconhecidas pela equipe de saúde da APS, planejando de forma integral e mais resolutiva o cuidado a essa pessoa e família.

Os profissionais participantes deste estudo, em sua maioria, se destacam neste cenário demonstrando uma visão ampliada de saúde, considerando todas as dimensões que envolvem o usuário no momento do atendimento, não focando apenas na lesão de pele.

São inúmeros os desafios para a prática da integralidade no cuidado a usuários com lesão de pele e que, ainda, há um distanciamento da teoria com as práticas na APS. Neste 
contexto cabe a reflexão aos profissionais de enfermagem, principalmente aos enfermeiros, em relação ao seu processo de trabalho, reconhecimento e apropriação deste cuidado e das repercussões deste na integralidade do cuidado e na consequente resolutividades das ações dele advindas. Evidentemente que essa aproximação inicial ao tema possui caráter exploratório e conta com alguns vieses importantes que precisam ser considerados no entendimento dos resultados (como por exemplo, a disparidade do grupo estudado, as diferenças entre contratos e regimes de trabalho, a desejabilidade em participar da pesquisa e as realidades contextuais diferenciadas de cada unidade). Mesmo assim, lançam um primeiro olhar sobre algo muito teorizado na elaboração dos processos de atenção e cuidado, mas ainda complexamente aplicável às estratégias de cuidado com caracteres mais procedimentais - como podem ser estas abordadas aqui, no tratamento de lesões de pele - ainda distanciadas e menos integradas a aspectos conceituais gerais do sistema de saúde brasileiro.

\section{REFERÊNCIAS}

1. Sakata KN, Mishima SM. Articulação das ações e interação dos Agentes Comunitários de Saúde na equipe de Saúde da Família. Rev Esc Enferm USP. 2012;46(3):665-72. https://doi.org/10.1590/ S0080-62342012000300019

2. VIII Conferência Nacional de Saúde. In: Anais da $8^{\underline{a}}$ Conf Nac de Saúde; 1986 Mar 17-21 [Internet]. Brasília; 1987 [citado 2017]. Disponível em: http://conselho.saude.gov.br/biblioteca/Relatorios/ relatorio_8.pdf

3. Brasil. Decreto 7508, de 28 de junho de 2011. Regulamenta a Lei no 8.080 , de 19 de setembro de 1990, para dispor sobre a organização do Sistema Único de Saúde - SUS, o planejamento da saúde, a assistência à saúde e a articulação interfederativa, e dá outras providências. DOU [Internet]. 2011 Jun 29 [citado 2017]. Disponível em: http://www.planalto.gov.br/ccivil_03/_ato20112014/2011/decreto/D7508.htm

4. Bonfada D, Cavalcante JRLP, Araujo DP, Guimarães J. A integralidade da atenção à saúde como eixo da organização tecnológica nos serviços. Ciênc Saúde Coletiva. 2012;7(2):555-60. https://doi. org/10.1590/S1413-81232012000200028

5. Gaiva M, Dias N, Siqueira V. Atenção ao neonato na estratégia saúde da família: avanços e desafios para atenção integral. Cogitare Enferm. 2012;17(4):730-7. https://doi.org/10.5380/ ce.v17i4.30382

6. Lira A, Sá J, Nogueira I, Medeiros M Fernandes M, Vitor A. Integralidade da pele em idosos: revisão da literatura segundo as cartas de promoção da saúde. Cogitare Enferm. 2012;17(4):767-74. https://doi.org/10.5380/ce.v17i4.30389

7. Dealey C. Cuidando de feridas: um guia para enfermeiras. $3^{\underline{a}}$ ed. São Paulo: Atheneu; 2013.

8. Hospital de Clínicas de Porto Alegre (HCPA). Avaliação e tratamento de feridas: orientações aos profissionais de saúde. Porto Alegre: HCPA/UFRGS; 2011.
9. Costa LM, Higino WJF, Leal FJ, Couto RC. Perfil clínico e sociodemográfico dos portadores de doença venosa crônica atendidos em centros de saúde de Maceió (AL). J Vasc Bras. 2012;11(2): 108-13. https://doi.org/10.1590/S1677-54492012000200007

10. Silva MH, Jesus MCP, Merighi MAB, Oliveira DM, Santos SMR, Vicente EJD. Manejo clínico de úlceras venosas na atenção primária à saúde. Acta Paul Enferm. 2012;25(3):329-33. https:// doi.org/10.1590/S0103-21002012000300002

11. Reis DB, Peres GA, Zuffi FB, Ferreira LA, Dal Poggetto MT. Cuidados às pessoas com úlcera venosa: percepção dos enfermeiros da estratégia de saúde da família. REME Rev Min Enferm. 2013;17(1):101-6.

12. Minayo ML, organizadora. Pesquisa social: teoria, método e criatividade. 30aㅡ ed. Petrópolis: Vozes; 2011.

13. Gray DE. Pesquisa no mundo real. 2aㅡ ed. Porto Alegre: Penso; 2012.

14. Ministério da Saúde (BR). Conselho Nacional de Saúde. Resolução 196, de 10 de outubro de 1996: diretrizes e normas regulamentadoras de pesquisa envolvendo seres humanos. Brasília; 1996.

15. Pizzinato A. Diversidad cultural y aprendizaje colaborativo: análisis del discurso docente. Liber. 2010;16(2):171-82.

16. Ferreira IRC; Vosgerau DSR; Moysés JS; Moysés ST. Diplomas Normativos do Programa Saúde na Escola: análise de conteúdo associada à ferramenta ATLAS TI. Cienc Saúde Coletiva. 2012;17(12):3385-98. https://doi.org/10.1590/S1413-81232012 001200023

17. Ministério da Saúde (BR). Departamento de Atenção Básica. Política Nacional da Atenção Básica. Brasília; 2011.

18. Ministério da Saúde (BR). Política Nacional de Humanização-PNH [Internet]. Brasília; 2013 [citado 2017]. Disponível em: http://bvsms. saude.gov.br/bvs/publicacoes/politica_nacional_humanizacao_pnh folheto.pdf

19. Marzari CK, Backes DS, Backes MS, Marchiori MT, Souza MT, Carpes AD. Realidade sociopolítica, ambiental e de saúde de famílias pertencentes a uma comunidade vulnerável. Cienc Saúde Coletiva. 2013;18(1):77-84. https://doi.org/10.1590/S141381232013000100009

20. Pinto IC, Marciliano CSM, Zacharias FCM, Stina APN, Passeri IAG, Bulgarelli AF. As práticas de enfermagem em um ambulatório na perspectiva da integralidade. Rev Latino-Am Enfermagem. 2012;20(5):909-16. https://doi.org/10.1590/S010411692012000500013

21. Silva LA, Casotti CA, Chaves SCL. A produção científica brasileira sobre a Estratégia Saúde da Família e a mudança no modelo de atenção. Cienc Saúde Coletiva. 2013;18(1):221-32. https://doi. org/10.1590/S1413-81232013000100023

22. Ogata $M N$, França Y. Atuação do auxiliar de enfermagem na Estratégia Saúde da Família. Acta Paul Enferm. 2010;23(4):506-11. https://doi.org/10.1590/S0103-21002010000400010

23. Zonta PM, De Arruda MP. O entendimento do usuário sobre processo de trabalho do enfermeiro da estratégia saúde da família. Rev Gepes Vida. 2015;1(2):71-85.

24. Oliveira BGRB, Nogueira GA, Carvalho MR, Abreu AM. Caracterização dos pacientes com úlcera venosa acompanhados no Ambulatório de Reparo de Feridas. Rev Eletr Enf. 2012;14(1): 156-63. https://doi.org/10.5216/ree.v14i1.10322 